\title{
Accelerated partial breast irradiation: 3-dimensional conformal external beam radiotherapy
}

\author{
Y. Hasan, F. Vicini \\ William Beaumont Hospital, Royal Oak, MI, USA.
}

\begin{abstract}
The use of three-dimensional conformal external beam accelerated partial breast irradiation (3D conformal $\mathrm{APBI}$ ) to deliver hypofractionated adjuvant radiation treatment to the region of the breast at highest risk for developing a local recurrence after lumpectomy is reviewed. Potential advantages of a 3D conformal radiation therapy approach for APBI compared to brachytherapy include improved dose homogeneity within the target volume and a non-invasive method for treatment delivery. Data from several early studies using 3D conformal APBI from New York University, William Beaumont Hospital, RTOG 0319, and Massachusetts General Hospital demonstrate technical feasibility, satisfactory early cosmetic outcome, and acceptable recurrence rates. To determine whether APBI provides equivalent local tumour control in the breast compared to conventional whole breast irradiation in the local management of early stage breast cancer, the first phase III randomized study of conventional whole breast irradiation vs. partial breast irradiation was opened conjointly by the NSABP and RTOG in March 2005. The results of this study will determine future directions in the treatment of early stage breast cancer, and, will help to define the role of APBI in breast conservation therapy.
\end{abstract}

Keywords: 3D conformal external beam radiotherapy; Accelerated partial breast irradiation; Breast conserving therapy

\section{Introduction}

The equivalence of breast-conserving therapy with surgery and adjuvant radiation therapy to mastectomy for the treatment of early stage breast cancer has conclusively been demonstrated in several phase III trials with long-term follow-up [1-3]. Accelerated partial breast irradiation (APBI) is an adjuvant treatment option for patients undergoing breast-conserving therapy for early stage breast cancer, who, due to co-morbid conditions, age, and/or

Correspondence to: Frank A. Vicini, MD, FACR, 3601 West Thirteen Mile Road, Royal Oak, MI 48073 6769, USA. E-mail: fvicini@beaumont.edu Tel: +1248 551 1219; Fax: +12485510089

Received:27/09/05

Accepted:21/11/05

First published online 17/02/06

$\mathrm{BCO} / 363 / 2005 / \mathrm{FO}$ logistics, are not suitable candidates for the standard course of 6-7 weeks of radiation therapy. APBI has historically been delivered via brachytherapy techniques employing multiple interstitial catheters [4] and, later, the MammoSite ${ }^{\mathrm{TM}}$ balloon applicator [5]. More recently, three-dimensional conformal external beam accelerated partial breast irradiation (3D conformal APBI) has allowed non-invasive delivery of an abbreviated course of adjuvant radiation treatment to the region of the breast at highest risk of developing local recurrence.

The potential advantages of a 3D conformal radiation therapy approach to partial-breast irradiation compared to brachytherapy include improved dose homogeneity within the target volume and, therefore, a likely better cosmetic outcome. In addition, elimination of an additional surgical procedure may reduce complication rates and cost. While brachytherapy 
requires additional training, most radiation facilities already have the technologic tools and experience required to deliver 3D conformal accelerated partialbreast treatment. The primary disadvantage is that the breast represents a moving target, and as a result, potentially larger volumes of normal breast tissue may need to be irradiated to avoid a geographic miss. At the present time, the two ways of delivering 3D conformal partial-breast irradiation differ primarily by patient positioning, either supine or prone. This review will discuss the major studies of 3D conformal accelerated partial-breast irradiation (Table 1), the technique of treatment delivery, potential challenges, and future directions.

\section{Rationale}

Data supporting the concept of partial-breast irradiation results from major randomized studies that have evaluated the role of standard, whole breast adjuvant radiation therapy in breast conservation $[2,6,7]$. These studies demonstrate that ipsilateral breast tumor recurrences largely occur at the original tumor bed. The ipsilateral breast 'elsewhere' failure (recurrences away from the tumor bed-generally considered new primary cancers) rate is similar to the contralateral breast new primary rate $(1.5-4 \%$ at 13 years) $[8,9]$. Based on these data, the partial breast target volume comprised of the lumpectomy cavity with a margin may be adequate in reducing the risk of local recurrence in women with small, adequately resected tumors. With a reduced target volume, hypofractionated radiation therapy, that is giving fewer total doses, but more radiation per dose, appears feasible. Goals are to reduce late toxicity, and to allow more women access to this treatment, since the time commitment becomes much less.

\section{History (studies utilizing external beam radiotherapy to deliver $\mathrm{PBI}$ )}

Polgar et al. reported the 5 year results of a phase I-Il study assessing adjuvant brachytherapy alone following breast-conserving therapy for stage I breast cancer [10], in which 45 patients with stage I breast cancer were prospectively selected to undergo adjuvant tumor bed radiotherapy (TBRT) via interstitial high dose rate (HDR) implants used to deliver either 4.33 Gy $\times 7$ fractions or 5.2 Gy $\times 7$ fractions. The 5year probability of cancer-specific, relapse-free and local recurrence-free survival was $90 \%, 85.9 \%$, and $95.6 \%$, respectively. Cosmetic results were excellent in $97.8 \%$ of patients and no toxicity greater than Grade 2 was observed. Based on the technical feasibility and results of the study, a phase III study was initiated and 126 further patients were randomized to receive 50 Gy whole brain radiation therapy (WBRT) $(n=63)$ or TBRT $(n=63)$ alone consisting of interstitial HDR brachytherapy delivering 5.2 Gy in 7 fractions $(n=46)$ or electron beam irradiation used to deliver 50 Gy $(n=17)$. At a mean follow-up of 30 months, locoregional control was $100 \%$ in both arms and the 3-year probability of cancer-specific and relapse-free survival rates were similar in both arms. Furthermore, radiation-related side effects were also not statistically different in the treatment arms.

The Christie Hospital, Manchester, United Kingdom, conducted the only other phase III prospective randomized trial comparing external-beam accelerated partial-breast irradiation (EB-APBI) to whole breast irradiation (WBI) [11,12]. The study included 708 patients with clinically palpable breast carcinomas and no palpable axillary adenopathy, who underwent lumpectomy (with no sentinel or axillary node dissection). They were randomized to receive either limited field (LF) partial-breast irradiation including the tumor bed, or wide field (WF) radiation including the whole breast and regional lymph nodes. In the 8-year update, overall survival rates were similar between the groups $(73 \%$ and $71 \%$ for the LF and WF groups, respectively). The actuarial breast recurrence rates were $20 \%$ and $11 \%$ in the LF and WF arms, respectively $(P=0.0008)$. However, when histology was factored in the analysis, invasive lobular histology appeared to account for a significant proportion of the local recurrences in the LF group compared to the WF group (34\% and $8 \%$, respectively). The local recurrence with invasive ductal carcinomas was similar in both arms (15\% with LF, and $11 \%$ with WF). Of note, the marginal miss/true recurrence (outside the treated field) of invasive ductal carcinoma in the LF arm was $5.5 \%$. The rate of fibrosis and telangiectasias was higher in the LF arm, with worse cosmetic outcome, as can be expected with APBI delivered via electron beams. Although the authors conclude that LF irradiation results in higher recurrence rate, $3 \mathrm{D}$ conformal $\mathrm{APBI}$ may have a significant role in the adjuvant treatment of early stage breast cancer when implementing appropriate patient selection and standard of care.

\section{Prone 3D conformal APBI}

Patient positioning during treatment delivery is geared toward optimizing daily reproducibility, limiting normal surrounding tissue dose, and ensuring appropriate dose coverage to the target structure. With the prone technique, patients who may benefit from the displacement of the lumpectomy cavity away from the chest wall, and thus, the heart and lungs, are those who are physically able to tolerate lying prone during simulation and treatment. The prone treatment position has been used to reduce breast tissue 
Table 1. Accelerated partial breast irradiation: EBRT studies [23]

EBRT: external beam radiotherapy; NYU: New York University; WBH: William Beaumont Hospital. 

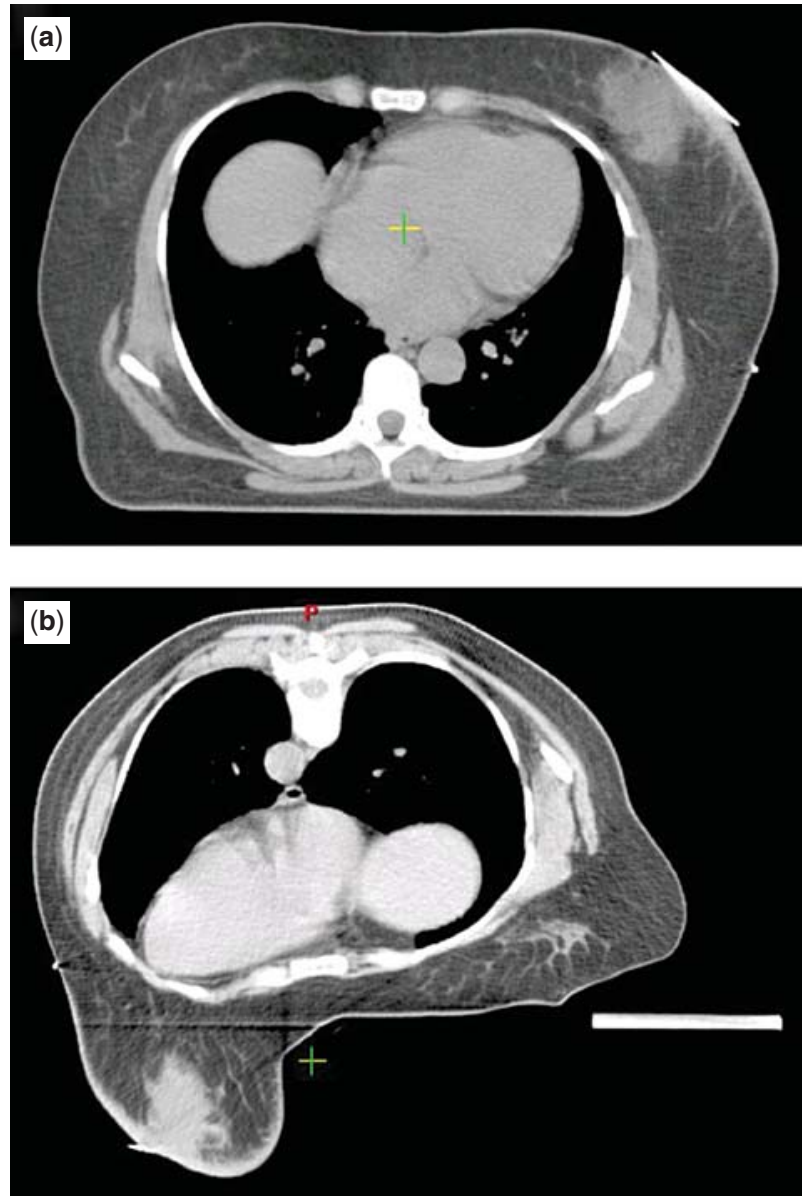

Figure 1.

Supine (A) and prone (B) patient postioning [15].

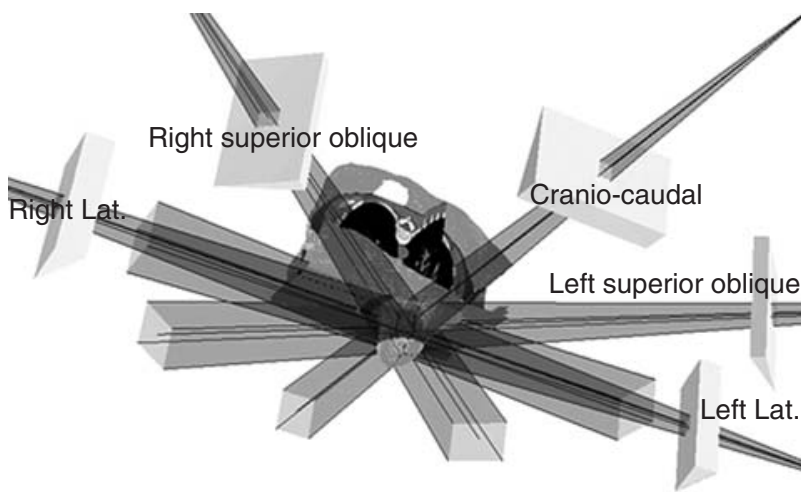

Figure 2.

Three-dimensional graphic reconstruction of five beam eye views for prone 3D conformal APBI [24].

motion resulting from cardiac systole and respiratory movement [13]. In such a position, excursion of the chest wall can be reduced to $5 \mathrm{~mm}$ [14], minimizing breast tissue motion and therefore target motion. Also, if the breast is allowed to hang through an opening in the table, this may allow the cavity to fall away from the chest wall due to gravity [15] (Figs. 1 and 2) and results in exclusion of the heart and lung from the treatment field [16].

\section{Clinical results}

\section{Phase I/II Trial of Prone 3D Conformal APBI - New York University}

On the basis of the results of a pilot study, Formenti et al. conducted a study of 47 post-menopausal women with stage I T1NO breast cancer, who were treated to $30 \mathrm{~Gy}$ in 56 Gy fractions over 10 days [17]. Other eligibility criteria included negative margins by at least $5 \mathrm{~mm}$. The patients were treated in the prone position and the planning target volume (PTV) was defined as the lumpectomy cavity with a $1.5 \mathrm{~cm}$ margin, with limitations anteriorly by skin and posteriorly by the chest wall. Computed tomography (CT)defined target volumes were treated with opposed mini-tangents with wedges. Fifty percent of the ipsilateral breast volume (BV) received less than $50 \%$ of the prescribed dose (PD). The contralateral breast and ipsilateral heart and lung were avoided completely in the beam arrangement. At a median follow-up of 18 months, no patients had local recurrence. Sixty percent of patients had Grade 1-2 erythema, which was the most common acute toxicity. Late toxicity was primarily Grade 1 and cosmetic results were mostly 'good' to 'excellent'. Only two patients had 'fair' cosmetic results and no patients had a worse score after radiation when compared to their postoperative baseline score.

\section{The William Beaumont Hospital experience - $3 D$ conformal $A P B I$ in supine position}

Initial clinical experience at William Beaumont Hospital (WBH) in utilizing 3D conformal radiation therapy to deliver partial-breast irradiation in patients with early stage breast cancer treated with breast-conserving therapy supported technical feasibility of such treatment delivery [18-20]. In this phase I/II study, 23 patients were prospectively enrolled between August 2000 and December 2002. An additional five patients were treated according to the guidelines of the protocol for compassionate purposes. Eligibility for the protocol included patient age $\geqslant 50$, tumor size $\leqslant 3 \mathrm{~cm}$, invasive ductal histology, lumpectomy with negative surgical margins by at least $2 \mathrm{~mm}$, negative axillary lymph nodes with a minimum of six sampled (or negative sentinel lymph node biopsy), and no extensive intraductal component. All patients underwent virtual CT breast simulation in the supine position. The clinical target volume (CTV) was defined as the lumpectomy cavity uniformly expanded by $10-15 \mathrm{~mm}$, limited by $5 \mathrm{~mm}$ from the skin surface 
Table 2. Dosimetric findings: CTV, PTV, and ipsilateral breast (protocol patients $[n=26]$ ) WBH.

\begin{tabular}{llll}
\hline $\begin{array}{l}\text { Dosimetric } \\
\text { characteristics }\end{array}$ & $\begin{array}{l}\text { Mean } \\
\text { value(\%) }\end{array}$ & $\begin{array}{l}\text { Median } \\
\text { value(\%) }\end{array}$ & Range(\%) \\
\hline $\begin{array}{l}\text { Maximum dose (\% of PD) } \\
\text { CTV coverage }\end{array}$ & 109 & 109 & $100-112$ \\
100\% IDL & 98 & 100 & $54-100$ \\
$95 \%$ IDL & 100 & 100 & $99-100$ \\
PTV coverage & & & \\
95\% IDL & 100 & 100 & $97-100$ \\
Ipsilateral breast & & & \\
coverage & & & \\
100\% IDL & 23 & 21 & $14-39$ \\
$75 \%$ IDL & 36 & 35 & $26-53$ \\
50\% IDL & 47 & 46 & $34-60$ \\
25\% IDL & 60 & 60 & $39-92$ \\
PTV/total breast volume & 17 & 17 & $11-22$ \\
\hline
\end{tabular}

CTV: clinical target volume; PTV: planning target volume; PD: prescribed dose; IDL: isodose line.

and lung-chest wall interface. PTV was defined by adding to the CTV $5 \mathrm{~mm}$ for breathing motion and another $5 \mathrm{~mm}$ for set-up error. Beam arrangement included 3, 4, 5, or 7 non-coplanar beams with $6 \mathrm{MV}$ photons alone in most patients. The initial dosefractionation schedule was 34 Gy delivered in 10 fractions of $3.4 \mathrm{~Gy}$ administered twice daily over 5 consecutive days with at least a 6-h interfraction interval, as in the Radiation Therapy Oncology Group (RTOG) 95-17 brachytherapy dose schedule. After treating six patients, the fraction size was increased to $3.85 \mathrm{~Gy}$, giving a total dose to $38.5 \mathrm{~Gy}$.

The heart and lung dose-volume histograms (DVHs) were below that for whole breast tangents for leftsided lesions. In all patients, a comparison was made in the doses delivered to normal tissues between the 3D conformal APBI plan and standard tangents. The goals were to accept plans that matched or preferably reduced doses to the heart and lung. Mean and median values (as well as ranges) for doses to the CTV, PTV, heart, and lung with the 3D conformal APBI plans were calculated and the dosimetric finding are summarized in Table 2. The mean and median coverage of the PTV by the $95 \%$ isodose line (IDL) was $100 \%$. The mean and median volume of the ipsilateral breast receiving $100 \%$ of the PD was $23 \%$ and $21 \%$, respectively. For all parameters examined, unnecessary doses to the heart and lung delivered with the APBI technique were less than equal to those delivered with standard WBI. Patients were initially seen in follow-up 4-6 weeks after completing treatment and then at 3-month intervals. The median follow-up duration was 8 months and cosmetic results and acute toxicity were assessed, revealing that $68 \%$ experienced Grade I toxicity and $11 \%$ had
Grade II in the first 6 weeks of follow up. Cosmetic results were rated as good/excellent in all evaluable patients at $6,12,18$, and $>18$ months after treatment. 6-month follow-up mammograms were negative in all evaluable patients $(n=12)$.

\section{Ongoing William Beaumont Hospital experience}

The ongoing WBH experience has been reported by Vicini et al. regarding 31 patients treated with $3 \mathrm{D}$ conformal APBI (20). The CTV consisted of the lumpectomy cavity plus a $10 \mathrm{~mm}$ or $15 \mathrm{~mm}$ margin, and the PTV consisted of the CTV plus a $10 \mathrm{~mm}$ margin for breathing motion and treatment set-up uncertainties. The PD was 34 or 38.5 Gy in 10 fractions b.i.d. separated by 6 hours and delivered in 5 consecutive days. Patients were treated in the supine position with 3-5 beams (mean 4) designed to irradiate the CTV with $<10 \%$ inhomogeneity and a comparable or lower dose to the heart, lung, and contralateral breast compared with standard wholebreast tangents. The mean coverage of the PTV by the $95 \%$ IDL was $100 \%$ (range: $97-100 \%$ ). The mean percentage of the breast receiving $100 \%$ of the PD was 23\% (range: 14-39\%). The median followup duration was 10 months (range: 1-30 months). While all patients had none to minimal skin changes during treatment, at the initial 6-week follow-up, $61 \%$ had Grade I toxicity and $10 \%$ had Grade II toxicity. The remaining $29 \%$ of patients had no observable side effects and no Grade 3 toxicities were observed. Cosmetic results were rated as good/excellent in all evaluable patients at $6,12,18$ months, and at $>2$ years after treatment. Based on these results, further studies were conducted including RTOG 0319.

\section{RTOG 0319 - Preliminary results}

Activated in August of 2003, the RTOG 0319 study was based upon the WBH experience. The same eligibility criteria and treatment technique, doses and fractionation schedule in RTOG 95-13 were employed in this study. The accrual goal was 42 patients and a total of 52 were treated by the completion of accrual in April 2004. Only 4 of the first 42 evaluable treatments were scored as unacceptable and the treatment technique was shown to be reproducible, as presented in the 2004 San Antonio Breast Cancer Symposium.

\section{Massachusetts General Hospital experience}

The initial clinical data acquired from the first 22 patients who underwent similar treatment in Boston, reported by Taghian et al. at 1-6 month follow-up, 
supports the feasibility and minimal acute toxicity with 3D conformal APBI demonstrated in other studies. The eligibility criteria included histology of invasive ductal carcinoma $\leqslant 2 \mathrm{~cm}$, negative lymph nodes, negative margins by at least $2 \mathrm{~mm}$, and no lymphovascular space invasion or extensive intraductal component. The PD was 32 Gy in 8 fractions b.i.d. separated by 6 hours, delivered over $4-5$ days. The PTV consisted of the lumpectomy cavity with a $15-20 \mathrm{~mm}$ margin. The dose inhomogeniety was less than $10 \%$ across the PTV. The patients were treated in the supine position with 3-4 beams of mostly mixed photons and electrons. The mean doses received by $20 \%$ (V20), 10\% (V10), and 5\% (V5) of the ipsilateral lung volumes were $2.3 \mathrm{~Gy}, 4.5 \mathrm{~Gy}$ and 6.7 Gy, respectively. The mean V20, V10, and V5 of the heart for left-sided lesions 1.5 Gy, 2.2 Gy, and 3.2 Gy, respectively. Fifty percent of the non-target BV was an average of 6.7 Gy. At the initial follow-up, $41 \%$ of patients had mild erythema and $9 \%$ had moderate erythema, with no patients having moist desquamation. Cosmetic results were good to excellent in all patients.

\section{Challenges and limiting factors in the application of $3 D$ conformal $A P B I$}

A primary potential disadvantage of $3 \mathrm{D}$ conformal APBI relates to organ motion effects and patient set up, which can necessitate a larger target volume in order to avoid a geographic miss. Based on previously published data [21], a $5 \mathrm{~mm}$ CTV to PTV expansion should account for normal breathing [18] and the use of $10 \mathrm{~mm}$ CTV to PTV margin also accounts for random and systematic components of set-up error. The final component of geometric uncertainty is the potential for the lumpectomy cavity to change shape and/or position independently of the surrounding breast tissue. A potential method of accounting for this motion involves on-line image guidance, which may employ the use of surgical clips to serve as a surrogate for the lumpectomy cavity [22] during the abbreviated course of treatment. The WBH data was analyzed to determine if certain variables could be identified to predict whether a patient was technically suitable for the 3D conformal quadrant technique [20]. Based on previously published PBI brachytherapy data, a 'borderline acceptable' plan was determined to have $>50-60 \%$ of the BV covered by the $50 \%$ IDL. Based on this endpoint, several factors were analyzed for their association with the probability of a particular case being appropriate for 3D conformal $\mathrm{APBI}$, including cavity volume, CTV volume, PTV volume, BV, CTV:BV ratio, PTV:BV ratio tumor location, etc. The factor found to have the highest correlation with the ability to meet the dose-volume constraints was the PTV:BV ratio, with ratios $>0.2$ unlikely to meet the requirements of the protocol. Finally, as with the delivery of any form of irradiation, the issue of verification of treatment delivery, when the uncertainty factors have been accounted for in planning, must also be addressed. This is especially important during EB-APBI as small inaccuracies may be more clinically significant resulting in potential geographic miss. As described previously, surgical clips have been used to delineate the lumpectomy cavity and this may be assessed at some institutions via CT scanning.

\section{Future directions}

To determine whether partial-breast irradiation limited to the region of the tumor bed following lumpectomy provides equivalent local tumor control in the breast compared to conventional WBI in the local management of early stage breast cancer, the first phase III randomized study of conventional WBI vs. partial breast irradiation (PBI) was opened conjointly by the National Surgical Adjuvant Breast and Bowel Project (NSABP) and RTOG in March 2005. This study includes patients with stage 0 , I, or II breast cancer resected by lumpectomy with tumor size $\leqslant 3 \mathrm{~cm}$ and no more than three histologically positive axillary lymph nodes. The stratification of patients is based upon disease stage (ductal carcinoma in situ (DCIS) only; invasive and node negative; invasive with 1-3 lymph nodes involved), menopausal status, hormone receptor status, and intention to receive chemotherapy. Randomization is completed after the patient is determined to be an appropriate candidate for possible APBI based on CT criteria including lumpectomy cavity shape, absolute volume, volume in reference to the whole breast volume, location, and distance from the skin surface. If the patient is determined to be appropriate candidate, randomization places her into either Group 1 (WBI) or Group 2 (PBI). WBI involves the delivery of 45-50 Gy in 25 fractions of 1.8-2.0 Gy per fraction to the whole breast followed by an optional boost to $\geqslant 60 \mathrm{~Gy}$. If the patient is randomized to Group 2, she will receive, as determined by her physicians in addition to patient preference, APBI via either of three modalities. The first two methods involve delivery of 34 Gy in 3.4 Gy fractions b.i.d. over 5-10 days using multi-catheter brachytherapy or the MammoSite balloon applicator. The third method of APBI delivery is via 3D conformal external-beam irradiation in which $38.5 \mathrm{~Gy}$ is delivered b.i.d. over $5-10$ days in 3.85 Gy fractions. The interfraction time for all treatments is at least 6 hours. The results of this study will determine future directions in the treatment of early stage breast cancer, and, will help to define the role of $\mathrm{APBI}$ in breast conservation therapy. 


\section{References}

1. Veronesi U, Luini A, Del Vecchio M, et al. Radiotherapy after breast-preserving surgery in women with localized cancer of the breast. N Eng J Med 1993; 328: 1587-1591.

2. Veronesi U, Marubini E, Mariani L, et al. Radiotherapy after breast-conserving surgery in small breast carcinoma: long-term results of a randomized trial. Ann Oncol 2001; 12: 997-1003.

3. Fisher B. Clinical trials for the evaluation of cancer therapy. Cancer 1984; 54: 2609-2617.

4. Vicini F, Baglan K, Kestin L, et al. The emerging role of brachytherapy in the management of patients with breast cancer. Semin Radiat Oncol 2002; 12: 31-39.

5. Dowlatshahi K, Snider HC, Gittleman MA, et al. Early experience with balloon brachytherapy for breast cancer. Arch Surg 2004; 139: 603-607.

6. Liljegren G, Holmberg L, Adami HO, et al. Sector resection with or without postoperative radiotherapy for stage I breast cancer: five-year results of a randomized trial. Uppsala-Orebro Breast Cancer Study Group. J Natl Cancer Inst 1994; 86: 717-722.

7. Clark RM, Whelan T, Levine M, et al. Randomized clinical trial of breast irradiation following lumpectomy and axillary dissection for node-negative breast cancer: an update. Ontario Clinical Oncology Group. J Natl Cancer Inst 1996; 88: 1659-1664.

8. Vicini FA, Kestin LL, Goldstein NS. Defining the clinical target volume for patients with early-stage breast cancer treated with lumpectomy and accelerated partial breast irradiation: a pathologic analysis. Int $J$ Radiat Oncol Biol Phys 2004; 60: 722-730.

9. Perera F, Chisela F, Engel J, Venkatesan V. Method of localization and implantation of the lumpectomy site for high dose rate brachytherapy after conservative surgery for T1 and T2 breast cancer. Int J Radiat Oncol Biol Phys 1995; 31: 959-965.

10. Polgar C, Sulyok Z, Fodor J, et al. Sole brachytherapy of the tumor bed after conservative surgery for T1 breast cancer: five-year results of a phase I-II study and initial findings of a randomized phase III trial. J Surg Oncol 2002; 80: 121-128.

11. Ribeiro GG, Dunn G, Swindell R, Harris M, Banerjee SS. Conservation of the breast using two different radiotherapy techniques: interim report of a clinical trial. Clin Oncol (Ryo Coll Radiol) 1990; 2: 27-34.

12. Ribeiro GG, Magee B, Swindell R, Harris M, Banerjee SS. The Christie Hospital breast conservation trial: an update at 8 years from inception. Clin Oncol (Roy Coll Radiol) 1993; 5: 278-283.

13. el Fallah Al, Plantec MB, Ferrara KW. Ultrasonic measurement of breast tissue motion and the implications for velocity estimation. Ultrasound Med Biol 1997; 23: 1047-1057.

14. Jozsef G, Luxton G, Formenti SC. Application of radiosurgery principles to a target in the breast: a dosimetric study. Med Phys 2000; 27: 1005-1010.

15. Formenti SC. External-beam partial-breast irradiation. Semin Radiat Oncol 2005; 15: 92-99.

16. Griem KL, Fetherston P, Kuznetsova M, et al. Threedimensional photon dosimetry: a comparison of treatment of the intact breast in the supine and prone position. Int J Radiat Oncol Biol Phys 2003; 57: 891-899.

17. Formenti SC, Truong MT, Goldberg JD, et al. Prone accelerated partial breast irradiation after breast-conserving surgery: preliminary clinical results and dose-volume histogram analysis. Int $J$ Radiat Oncol Biol Phys 2004; 60: 493-504.

18. Baglan KL, Sharpe MB, Jaffray D, et al. Accelerated partial breast irradiation using 3D conformal radiation therapy (3D-CRT). Int J Radiat Oncol Biol Phys 2003; 55: 302-311.

19. Vicini F, Arthur D, Polgar C, Kuske R. Defining the efficacy of accelerated partial breast irradiation: the importance of proper patient selection, optimal quality assurance, and common sense. Int J Radiat Oncol Biol Phys 2003; 57: 1210-1213.

20. Vicini FA, Remouchamps V, Wallace M, et al. Ongoing clinical experience utilizing 3D conformal external beam radiotherapy to deliver partial-breast irradiation in patients with early-stage breast cancer treated with breast-conserving therapy. Int J Radiat Oncol Biol Phys 2003; 57: 1247-1253.

21. Frazier RC, Vicini FA, Sharpe MB, et al. Impact of breathing motion on whole breast radiotherapy: a dosimetric analysis using active breathing control. Int $J$ Radiat Oncol Biol Phys 2004; 58: 1041-1047.

22. Weed DW, Yan D, Martinez AA, et al. The validity of surgical clips as a radiographic surrogate for the lumpectomy cavity in image-guided accelerated partial breast irradiation. Int J Radiat Oncol Biol Phys 2004; 60: 484-492.

23. Rosenstein BS, Lymberis SC, Formenti SC. Biologic comparison of partial breast irradiation protocols. Int $\mathrm{J}$ Radiat Oncol Biol Phys 2004; 60: 1393-1404.

24. Formenti SC, Rosenstein B, Skinner KA, Jozsef G. T1 stage breast cancer: adjuvant hypofractionated conformal radiation therapy to tumor bed in selected postmenopausal breast cancer patients-pilot feasibility study. Radiology 2002; 222: 171-178. 